\title{
Pulmonary function testing in preschool children: from the lab to the clinical practice
}

Pulmonary function testing plays an unquestionable role both in the physiology and pathophysiology research of the respiratory system and the clinical approach of a patient with a respiratory disease. The respiratory system of young children is under continuous growth and development; therefore the correct interpretation of pulmonary function test results is a sensitive issue, where pathological processes should be differentiated from those related to expected physiological changes. The effects of prematurity on pulmonary function, bronchopulmonary dysplasia, viral infections and their consequences, and recurrent wheezing, are all areas of great interest under constant investigation in pediatrics.

Preschool children have a high prevalence of airway conditions, and the possibility of measuring pulmonary function in this group is particularly attractive. The presence of nonspecific symptoms, such as wheezing, and the difficulties posed when trying to measure them functionally, sometimes lead to a misdiagnosis. This situation favors the implementation of empiric treatments because such symptoms are wrongly compared to conditions with a different pathophysiology, e.g. asthma in older children.

Measurement is complicated by the characteristics of young children, who have a short attention span and difficulty to perform respiratory coordination and control maneuvers, which are critical in these tests.

If there was an ideal system to measure pulmonary function in preschool children, it would imply an easy and short maneuver that would require minimum collaboration from the patient, affordable and portable equipment, and most importantly, a safe system that can be used in all age groups leading to reliable, highly reproducible results that can be easily interpreted, providing a comprehensive assessment of the respiratory function.

Different efforts have been made over the years looking for the appropriate system for preschool children. Although the ideal system has not yet been found, the modification of software developed for adults and older children, the adaptation of techniques, the publication of test recommendations ${ }^{1}$ and reference values, ${ }^{2}$ have all contributed to the possibility of performing measurements in younger children.
At present, in this age group it is plausible to perform spirometries, measure lung volume by gas dilution, apply forced oscillation techniques and flow interrupter techniques for the measurement of airway resistance. Each of these methods has specific advantages and disadvantages, which should be weighed at the time of ordering a test and interpreting its results.

Airway resistance measurement by the interrupter technique (Rint) was first described in 1927, it was implemented in the 70s, and had a revival in the 2000s. During this latter period, different studies were published on its methodological characteristics, reference values and technical recommendations to perform it.

In spite of its long history, this measurement method is far from being widely used. The main controversy is quite likely related to the high variability among longitudinal measurements in the same patient, ${ }^{3}$ which renders the Rint less useful for the follow-up of these patients, most important in clinical practice. Likewise, due to aspects inherent to the method, the Rint may underestimate airway resistance in severe obstructive diseases. Besides, small changes in measurement conditions, such as the position of the head, cheek or floor of the mouth support, and the use or not of nose clips can substantially modify results. ${ }^{4}$

In the present issue of the Archivos Argentinos de Pediatría, Zuriarrain Reyna, et al., evaluate the applicability and reproducibility of the Rint in a representative sample of pediatric patients and compare results to those obtained with other methods to measure resistance. Authors show that acceptable and reproducible tests can be obtained in $92 \%$ of children, and in more than $70 \%$ of children under 7 years old, and that their results are adequately correlated with those obtained by other methods, and this is probably the most important contribution of this study. It should be noted that, as suggested under the discussion section, the different methods not necessarily measure the same physiological phenomenon. However, this type of tests is definitely the necessary path to follow to better understand preschool children physiology.

From a clinical standpoint, it is expected that pulmonary function testing will complement the information obtained from the case history 
and physical examination, help to establish a diagnosis, estimate the prognosis, and determine the course of the disease and the response to therapeutic interventions. However, unfortunately at present there is not enough evidence to support the use of routine pulmonary function tests in preschool children to monitor them and make decisions on how to approach them. For the time being, its usefulness is confined to epidemiological and research studies.

Verónica Aguerre, M.D.

Pediatric Pulmonologist

Division of Pulmonology

Hospital de Pediatría "Prof. Dr. Juan P. Garrahan" http:/ /dx.doi.org/10.5546/aap.2013.468

1. Beydon N, Davis SD, Lombardi E, Allen JL, et al. An oficial American Thoracic Society / European Respiratory Society statement: pulmonary function testing in preschool children. Am J RespirCrit Care Med 2007; 175: 1304-1345.

2. Stanojevic S, Wade A, Lum S, Stocks J. Reference equations for pulmonary function tests in preschool children: A Review. Pediatric Pulmonology 2007; 42: 962-972.

3. Chan EY, Bridge PD, Dundas I,PaoCS, Healy MJ, McKenzie SA. Thorax 2003; 58: 344-347.

4. Hadjikoumi I, Hassan A, Milner AD. Effects of respiratory timing and cheek support on resistance measurements, before and after bronchodilation in asthmatic children using the interrupter technique. Pediatric Pulmonology 2003; 36: 495-501.

\section{Rights and responsibilities of electronic health records (EHR) users caring for children}

The care of children and neonates presents complex challenges for the design and operation of healthcare facilities and EHRs worldwide. For clinicians to provide the highest quality, safe and effective care to children, EHRs providing care to children must be properly designed and configured and clinicians must use them correctly. Organizations that provide their clinicians with state-of-the-art EHRs and grant them the "professional rights" we previously identified along with these "pediatric amendments" could see dramatic improvements in clinician usage of their EHRs. This will lead us closer to the ultimate goal of improving the quality, safety, and effectiveness of care delivered to children.

Establishing a safe and effective electronic health record-enabled (EHR) healthcare delivery system is complex and challenging. In addition to support from executive leadership, a robust EHR from a reputable vendor, and access to knowledgeable and committed information technology professionals, clinician support is instrumental in overcoming the challenges. While there is an increasing breadth of knowledge about good clinical practices needed to address EHR implementation and use in the general population, clinicians responsible for the care of neonates, children, and adolescents face a unique set of additional challenges. For example, children have unique EHR requirements related to dosing of medications as well as specific needs related to their growth and development that the EHR needs to facilitate. ${ }^{1}$

In order to encourage a dialogue between clinicians and other stakeholders to help address and overcome these challenges, we previously proposed that front-line practicing clinicians be given certain "professional rights" for "must have" EHR features, functions, and user privileges that are critical to provide high quality and safe care. We also proposed that each "right" be accompanied by a corresponding user responsibility. Because of the unique circumstances involving the safe and effective care of children and that fact that most children are not cared for in facilities where the EHR has been designed exclusively for children, in this paper we propose "pediatric amendments" to our previously proposed "Rights and Responsibilities of Users of EHRs". ${ }^{2}$ All previously identified rights and responsibilities still apply along with these new pediatric-specific items discussed below.

Support for medication prescribing in children

The epidemiology of harm associated with medication prescribing for neonates and children is very different than adult patients. Both hospitalized and ambulatory patients are at higher risk of harm from drug dosing errors than from drug-drug interactions. ${ }^{3}$ Clinicians seeing pediatric patients have the right to both in patient 
and ambulatory electronic prescribing systems that are safer and more effective for children and include weight-based dosing recommendations, age appropriate dosing calculators, dose-range checking, and pediatric-specific drug-drug interaction alerts. ${ }^{4,5}$

Clinicians seeing pediatric patients have the responsibility to consistently and reliably document patient weights, and should maintain familiarity with medication dosing guidelines to mitigate the effect of automation bias. ${ }^{6}$

\section{Electronic display of growth charts}

Visual display of patient information is an important decision support tool. Clinicians should have the right to view their young patients'anthropometric data using growth charts that display age-based percentiles for weight, height, head circumference, and body mass index (BMI) within their EHR.?

All of these age-appropriate displays require up-to-date, accurate data capture; therefore, clinicians have the responsibility to record or facilitate the recording of patient's height, weight, and head circumference. Additionally, they should use this information to apply the appropriate age-specific clinical guidelines and provide copies of these charts to parents.

\section{Child-friendly, EHR-equipped exam room}

While not a specific feature or function of the EHR, clinicians caring for children have the right to an EHR-equipped exam room that is designed using appropriate human factors principles. For example, rooms should have a layout that provides adequate room for the patient, a parent and the clinician to move around. ${ }^{8}$ In addition, keyboards and touchscreens should be cleaned and disinfected on a regular basis. ${ }^{9}$ Finally, the computer, if wall-mounted, should be sturdy enough to withstand a child swinging from the support arm. Clinicians have the responsibility for positioning the monitor so that he/she, as well as the parent and the patient can see the screen simultaneously. This is particularly important in pediatrics, as children cannot rationalize the use of a computer in the exam room and may unintentionally misinterpret the intention.

\section{User interface that supports correct identification of patients}

Several studies have suggested that pediatric patients in general and neonates in particular are at higher risk for misidentification because of naming issues during the newborn period and siblings being treated simultaneously at pediatric visits. ${ }^{10}$ Clinicians who see these patients have a right to an EHR user interface which minimizes wrong-patient errors. Such functionality may include limiting users to one open chart at a time, availability of patient pictures within the EHR, and including additional patient verification processes with computerized order entry systems. ${ }^{11}$,

Electronic systems themselves may actually carry the unintended consequence of increasing the risk for wrong-patient errors. Users of these systems have a responsibility to ensure that processes are setup to capture patient photographs in the EHR, and that misidentification errors are appropriately reported and fixed.

\section{An EHR that supports adolescent confidentiality}

Although exact legal requirements vary, most countries acknowledge that adolescents have the right to keep mental, behavioral, and sexual healthcare confidential from their parents or guardians. Unfortunately, many commercial EMR's do not yet provide the functionality needed to respect these legal and ethical positions. ${ }^{12}$ Pediatric users have the right to EHR software which includes default settings for adolescent privacy, customizable point-of-care privacy controls for clinicians, clear on-screen labeling of confidential data elements, patientadjustable proxy access capabilities for patient portals, and suppression capabilities for specific items on post-visit summaries, bills, and postvisit surveys. In addition, adolescent privacy standards must be built into health information exchange data sharing agreements.

Clinicians seeing adolescent patients have the responsibility to understand local adolescent confidentiality regulatory requirements. They should also review the entire patient experience from registration to post-clinic surveys to ensure that the adolescent's confidentiality is maintained in light of these requirements.

\section{EHR content that supports pediatric practice}

To deliver appropriate preventative wellchild care, pediatricians have the right to an EHR with content that supports the care of children. This includes appropriate decision support rules for timely preventive care such as 
administration of immunizations and linkages to immunization registries as well as content for pediatric normative values (e.g. laboratory test values) that frequently change with age. ${ }^{13}$ Furthermore, EHRs must be optimized to support recording of quality measures for pediatrics. Pediatricians have the responsibility to review decision support rules (e.g. do they match local vaccination schedules) and record key data that would lead to the generation of appropriate decision support.

Dean F. Sittig, PHD

University of Texas School of Biomedical Informatics and the UT-Memorial Hermann Center for Healthcare Quality \& Safety, Houston, Texas, USA

Hardeep Singh, MD, MPH

Houston VA Health Sciences Research \& Development Center of Excellence, Michael E. DeBakey Veterans Affairs Medical Center and the Section of Health Services Research, Department of Medicine, Baylor College of Medicine, Houston, Texas, USA

Christopher A. Longhurst, MD, MS Division of Systems Medicine, Department of Pediatrics, Stanford University School of Medicine, Stanford, CA, USA

http:/ /dx.doi.org/10.5546/aap.2013.469

1. Spooner SA. Council on Clinical Information Technology, American Academy of Pediatrics. Special requirements of electronic health record systems in pediatrics. Pediatrics 2007;119(3):631-7.

2. Sittig DF, Singh H. Rights and responsibilities of users of electronic health records. CMAJ 2012;184(13):1479-83.

3. Kaushal R, Goldmann DA, Keohane CA, Christino M, et al. Adverse drug events in pediatric outpatients. Ambul Pediatr 2007;7(5):383-9.

4. Harper MB, Longhurst CA, McGuire T, et al. Core drugdrug interaction alerts for inclusion in pediatric electronic health records with computerized prescriber order entry. $J$ Patient Safety (2013 in press).

5. Stevens LA, Palma JP, Pander KK, Longhurst CA. Immunization registries in the EMR Era. Online J Public Health Inform 2013;5(2);1-11. [Consultado 24 de julio de 2013] Disponible en: http://ojphi.org/ojs/index.php/ ojphi/article/view/4696/3717

6. Goddard K, Roudsari A, Wyatt JC. Automation bias: a systematic review of frequency, effect mediators, and mitigators. I Am Med Inform Assoc 2012;19(1):121-7.

7. Lowry S, Quinn M, Ramaiah M, Brick D, et al. A Human factors guide to enhance EHR usability of critical user interactions when supporting pediatric patient care. National Institutes of Standards and Technology: US Department of Commerce.06/28/2012. NISTIR 7865. [Consultado: 24 de julio de 2013 ] Disponible en: http:// www.nist.gov/healthcare/usability/upload/NISTIR-7865.pdf

8. Henriksen K, Dayton E, Keyes MA, Carayon P, Hughes R. Understanding adverse events: a human factors framework. En:Hughes RG(ed). PatientSafety and Quality: An evidence-based handbook for nurses. Rockville (MD): Agency for Healthcare Research and Quality (US); 2008 Apr.Disponibleen:http://www.ncbi.nlm.nih.gov/books/ NBK2666.

9. Neely AN, Sittig DF. Basic microbiologic and infection control information to reduce the potential transmission of pathogens to patients via computer hardware. J Am Med Inform Assoc 2002;9(5):500-8.

10. Gray JE, Suresh G, Ursprung R, Edwards WH, et al. Patient misidentification in the neonatal intensive care unit: quantification of risk. Pediatrics 2006 ;117(1):e43-7.

11. McCoy AB, Wright A, Kahn MG, ShapiroJS, et al. Matching identifiers in electronic health records: implications for duplicate records and patient safety. BMJ Qual Saf 2013;22(3):219-24.

12. Anoshiravani A, Gaskin GL, Groshek MR, Kuelbs C, Longhurst CA. Special requirements for electronic medical records in adolescent medicine. J Adolesc Health 2012;51(5):409-14.

13. SpoonerSA, Classen DC. Data standards and improvement of quality and safety in child healthcare. Pediatrics 2009;123(Suppl 2):S74-9. 